\title{
Chemical Engineering Journal, DOI: 10.1016/j.cej.2018.07.214 Hydrodynamics of a packed bed of non-spherical polydisperse particles: a fully virtual approach validated by experiments
}

\author{
Victor Pozzobon, Julien Colin \& Patrick Perré \\ LGPM, CentraleSupélec, Université Paris-Saclay, SFR Condorcet FR CNRS 3417, \\ Centre Européen de Biotechnologie et de Bioéconomie (CEBB), \\ 3 rue des Rouges Terres 51110 Pomacle, France
}

\begin{abstract}
This work presents a numerical workflow to generate a virtual packed bed made of non-spherical polydisperse particles, and subsequently predict its permeability. Wood chips were taken as an illustration. First, chips are sized before being recreated numerically. Then, using LMGC90, a DEM code, a packed bed made of those chips was generated. Once bed internal had been sampled, CFD tools belonging to the OpenFOAM library were used to mesh the geometry (snappyHexMesh) and compute fluid motion (simpleFoam). Finally, using numerical results, the bed permeability was computed in both Stokes and inertial regimes - turbulence being described by Launder-Reece-Rodi model. In parallel, experimental measurements of the permeability of a packed bed, made of the exact same wood chips, was carried out. These experiments were used as a reference to challenge numerical results. The permeability value delivered by the workflow is $16.0 \%$ higher than the experimental value. This value has to be compared with Kozeny-Carman equation estimation which overestimates bed permeability by $115 \%$. Going one step further, this framework was successfully used to compute inertial effects constant of the Forshheimer equation for our packed bed. Throughout this article, a special care has been taken in explaining and evaluating the impact of all the key parameters, namely, number of particles that have to be sized, mesh refinement level, numerical domain dimensions. This workflow opens the door to numerical estimation of bed tortuosity, dispersion coefficients, volumetric heat exchange coefficients, and much more, using the particle size distribution as unique input data.

Keywords: Granular media, Porous media, Permeability, DEM, CFD, OpenFOAM
\end{abstract}

\section{Introduction}

Nowadays, chemical engineering heavy relies on packed bed reactors. Most of the time, these beds are made of particles poured into a container which is then crossed by a reacting flow $[1,2,3,4]$. It is widely admitted that the hydrodymic properties permeability, tortuosity, dispersion coefficients, ... - of such devices are key to properly operate them $([5,6,7,8,9,10])$. Yet, they can be quite hard determine. Among them, permeability is of key importance as it directly influences the pressure drop across the bed, hence the pumping cost.

Three different approaches are available the determine this parameter. The first one is to use correlations coming from the literature such as Ergun [11] or Kozeny-Carman expression [12]. These semi-empirical correlations are widespread. They were derived, most of the time for packed bed made of monodisperse spheres. Even though, they can present refinements taking into account media made of non-spherical particles, polydispersed media and inertial effects, they usually only yield an estimation of the permeability.

The second method consists in experimentally measuring the permeability value. The first experiments were carried out by Darcy [13] (Eq. 1) who introduced the concept of permeability for porous media.

$$
\frac{Q}{S}=\frac{\kappa}{\mu} \frac{\Delta P}{h}
$$

This equation balances the most important parameters of the problem at stake. As fluid flows through a porous medium, it flows all the more rapidly that the pressure gradient is high, the medium is permeable and the fluid is close to being inviscid. Today, permeability measurements are quite common in the literature $[14,15,16,17,18]$. Usually, they consist in measuring, in steady state, a pressure drop over a bed crossed by a fluid of known viscosity under a well controlled flow rate (Eq. 2).

$$
\kappa=\frac{Q \mu h}{S\left(P_{\text {in }}-P_{\text {out }}\right)}
$$

Yet, these measurements are not always easily conducted. Indeed, the flow has to reach steady state, which may take a tremendous amount of time for almost not permeable media, such as rocks [19] or tropical wood species [20]. The other 
extreme is very permeable media, that would induce only a minor pressure drop. In this case, the experimental apparatus has to be long enough so that a pressure drop can be precisely measured. Another technique is to use liquids instead of gases [21], as they have a higher viscosity. The drawback is that liquids are less convenient to use than gas namely because it is very difficult to ensure full saturation of the sample and to avoid degasing during measurement.

The last approach consists in using a numerical tool to assess for the physical properties. First, the medium is scanned [22]. Then, the void inside the solid matrix is meshed. Finally, using a Computational Fluid Dynamic - CFD - software, Navier-Stokes or only Stokes (when inertial effects are neglected, Eq. 3) equations are computed in-between the solids in order to yield the fluid motion [23, 24, 25]. Then, the pressure drop across the numerical model is extracted and used to compute the permeability value. In addition, using a numerical approach, it is possible to obtain the different component of the permeability tensor [25]. From a more general perspective, this kind of numerical approach is spreading fast to different fields of science, such the design of heat exchanger [26, 27, 28] or static mixer [29, 30].

$$
R e=\frac{\rho \overline{d_{e q}}|\vec{u}|}{\mu} \lesssim 1
$$

The two last approaches are the most reliable, yet they require time and high quality materials: a permeability measurement apparatus for the experimental one, a 3D scanner for the last one. Furthermore, the fully digital approach suffers a drawback compared the experimental one: as a result of Poiseuille law, the smallest pores contributes mostly to the fluid resistance. Consequently, the 3D morphological description should be accurate enough to capture correctly the geometry of the smallest pores along the fluid pathways.

The objective of this article is to propose the robust, fully numerical workflow capable to generate and characterize a packed bed.

The objective of this work is to propose a robust, fully numerical workflow allowing to, firstly, generate and, secondly, characterize packed bed hydrodynamics. This numerical methodology could be applied enhance reactor design, for example by reducing pumping costs, or understanding channeling origin and consequently preventing it. This tool could also be used to diagnose problems on existing reactors, for example, where tortuosity distribution leads to inhomogeneous products. To illustrate this workflow, we choose to work on wood chips beds as biomass thermochemical conversion is becoming increasingly popular [31, 32, 33, 34]. Yet, wood chips packed beds hydrodynamic properties are still scarce in the literature. In this challenging case, the proposed tool is capable of computing the permeability of polydisperse granular bed made of nonspherical particles. In details, the numerical workflow is constituted of few basic steps. First, particles are sized and reproduced numerically. Then, using DEM and CFD codes, a granular bed is created and fluid flow inside of it is computed. In parallel, experimental measurements were conducted in order to assess for the reliability of the workflow's prediction.

Throughout this article, a special care will been taken in explaining and evaluating the impact of all the key numerical parameters. Finally, deviation between the three available approaches is assessed, taking the experimental value as reference.

\section{Nomenclature}

\begin{tabular}{ll} 
Latin symbols & \\
\hline$C$ & inertial constant, - \\
$d$ & diameter, $\mathrm{m}$ \\
$\vec{f}$ & body forces, $\mathrm{N}$ \\
$\vec{g}$ & acceleration due to gravity, $\mathrm{m}^{2} / \mathrm{s}$ \\
$h$ & height, $\mathrm{m}$ \\
$M$ & molar mass, $\mathrm{g} / \mathrm{mol}$ \\
$\vec{n}$ & normal vector, - \\
$P$ & relative pressure, $\mathrm{Pa}$ \\
$Q$ & volumetric flow rate, $\mathrm{m}^{3} / \mathrm{s}$ \\
$S$ & surface, $\mathrm{m}^{2}$ \\
$\vec{u}$ & macroscopic velocity, $\mathrm{m} / \mathrm{s}$ \\
$V$ & volume, $\mathrm{m}^{3}$ \\
& \\
\hline Greek symbols & \\
\hline$\alpha$ & Ergun equation Stokes regime constant, - \\
$\beta$ & Ergun equation inertial regime constant, - \\
$\Delta$ & difference operator, - \\
$\epsilon$ & porosity, - \\
$\kappa$ & permeability, $\mathrm{m}^{2}$ \\
$\mu$ & dynamic viscosity, Pa.s \\
$\rho$ & density, kg/m $\mathrm{m}^{3}$ \\
$\bar{\Sigma}$ & stress tensor, Pa \\
$\sigma$ & surface tension, $\mathrm{N} / \mathrm{m}$ \\
& sphericity, -
\end{tabular}




\begin{tabular}{ll} 
Subscripts & \\
\hline bed & bed \\
$e q$ & equivalent \\
in & inlet \\
mesh & mesh \\
out & outlet \\
ref & reference \\
$w-w$ & wood over wood \\
$w-p v c$ & wood over PVC \\
Other symbols \\
\hline$\nabla$ & nabla operator \\
$|\vec{a}|$ & norm \\
$\bar{a}$ & arithmetic average \\
$\overline{\bar{A}}$ & tensor
\end{tabular}

\section{Granular medium: wood chips}

Beech (Fagus sylvatica) wood chips (Fig. 1a) were used to illustrate this workflow. They were supplied by S.P.P.S. (Argenteuil, France). Even thought they exhibit a large range of shape and size, wood chips can be considered as rough parallelepipeds. Their granulometry should be ranging between 4.5 and $9.0 \times 10^{-3} \mathrm{~m}$ according the supplier data. The moisture content was measured and is equal to $11.6 \%$ (weight dry basis). In order to reproduce the chips numerically, several options were available: using a 3D scanner, using photography and image treatment (yields only 2D informations), or measuring them with a caliper [35]. For the sake robustness of the propose method and ease to employ, we choose to use a caliper $\left(0.1 \times 10^{-3} \mathrm{~m}\right.$ precision $)$ and measure manually the dimensions of the chips in the three directions. This method is rather fast (1h38 for 536 chips). Its main drawback is that it requires to assume that the chips are parallelepipeds.

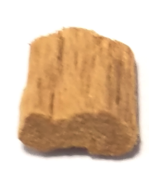

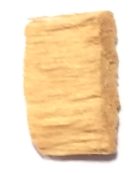

(a)
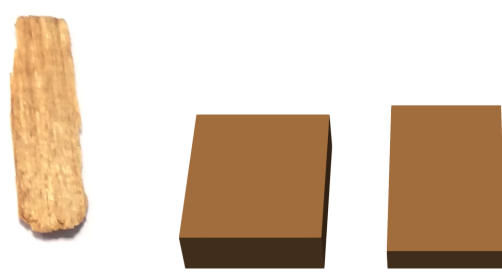

(b)

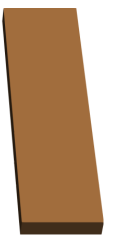

Figure 1: Example of actual wood chips (a) and their numerical equivalents (b)

We used the diameter of the volume-equivalent sphere (as defined in Eq. 4) as chip characteristic length. It was calculated for each of the 536 chips. Indeed, knowing their length $\times$ height $\times$ width $(\mathrm{L} \times \mathrm{H} \times \mathrm{W})$, assuming a parallelepipedic shape, we can compute the surface area $\mathrm{A}=2 \times(\mathrm{L} \times \mathrm{W}+\mathrm{W} \times \mathrm{H}+\mathrm{W} \times \mathrm{L})$ and volume: $\mathrm{V}=\mathrm{L} \times \mathrm{H} \times \mathrm{W}$ of each chip. Thus, it is possible to later compute several quantities if interest. Once averaged over the distribution, the equivalent diameter is equal to the Sauter mean diameter.

$$
d_{e q}=\frac{6 V}{S}
$$

In our case, the third method was chosen. Special care was taken in sampling the chips. Two factors may bias the sampling. The first one is known as kinetic sieving [36, 37], in short, when shaking an polydisperse granular medium, the larger particles have a propensity to rise in the container. Hence, sampling only the top of the container would lead to an overestimation of the particles sizes. To avoid such an effect, wood chips were poured from one container to the other several times before sampling. In addition, the container was sampled at different height.

The second factor arises from the natural tendency of the experimenter to measure the larger particles first. Figure 2a reports the averaged equivalent diameter of 536 chips ordered per measurement date. One can see that the average decreases with the number of measured chips. It means that, unconscously, the larger particles were sized first. So, to be as accurate as possible, all the sampled chips have to be measured, even though one could consider that few hundreds are enough. Furthermore, even for a large number of measured chips, the averaged equivalent diameter does not seem to converge (Fig. 2a). This would mean that an even higher amount of chips should be measured. Yet, one should keep in mind that, here, the average operator bears meaning when applied to random variables. In this very case, given the fact that larger chips are sized first, the chip diameter can not considered as a random variable. Hence, before computing the average and the standard deviation of this quantity, the diameter list has to be shuffled. Figure $2 \mathrm{~b}$ reports the averaged equivalent diameter computed 
with the shuffled list of diameter. From this graph, one can see that the averaged equivalent diameter converges after about 50 measurements, while its standard deviation stabilizes after 250 measurements.

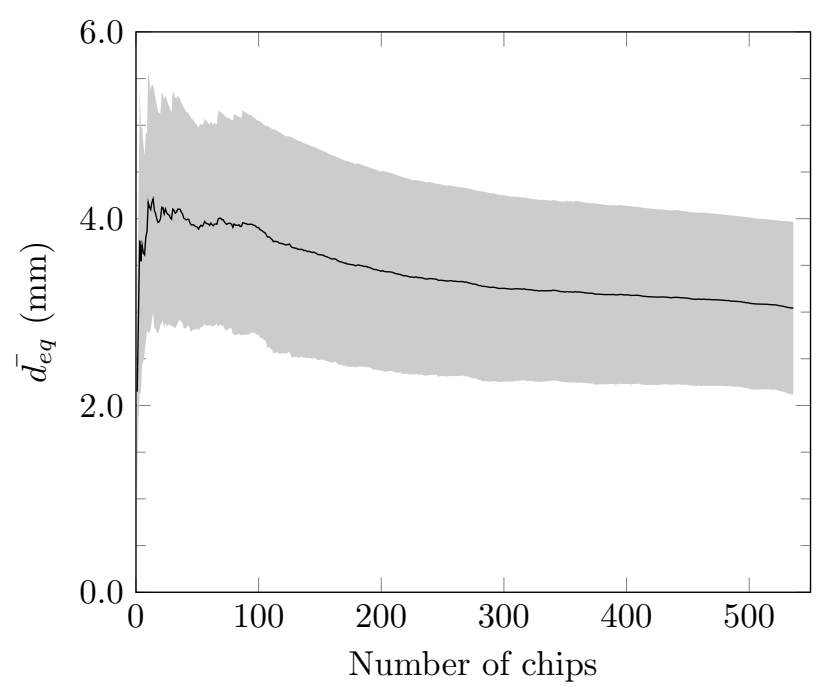

(a)

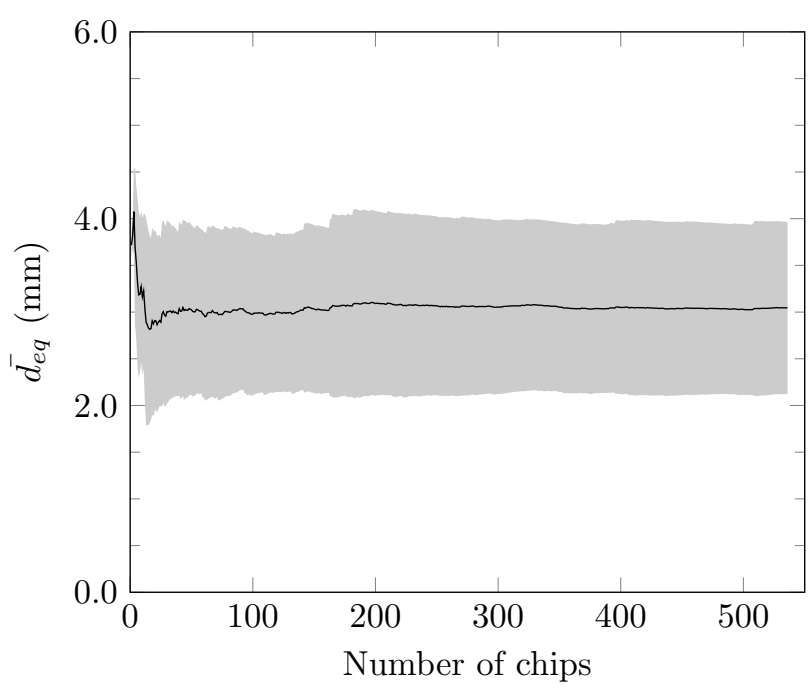

(b)

Figure 2: Averaged chip equivalent diameter versus number of measured chips. (a) Ordered per measurement date. (b) Shuffled. Black line: averaged value, gray area: \pm 1 standard deviation

Finally, one last caution that has to be taken is to verify that the equivalent diameters distribution is monomodal. Figure 3 reports the equivalent diameters distribution of the 536 measured chips. As one can see, it exhibits a clear monomodal distribution. Hence it is possible to derive from it an averaged value that bears actual meaning.

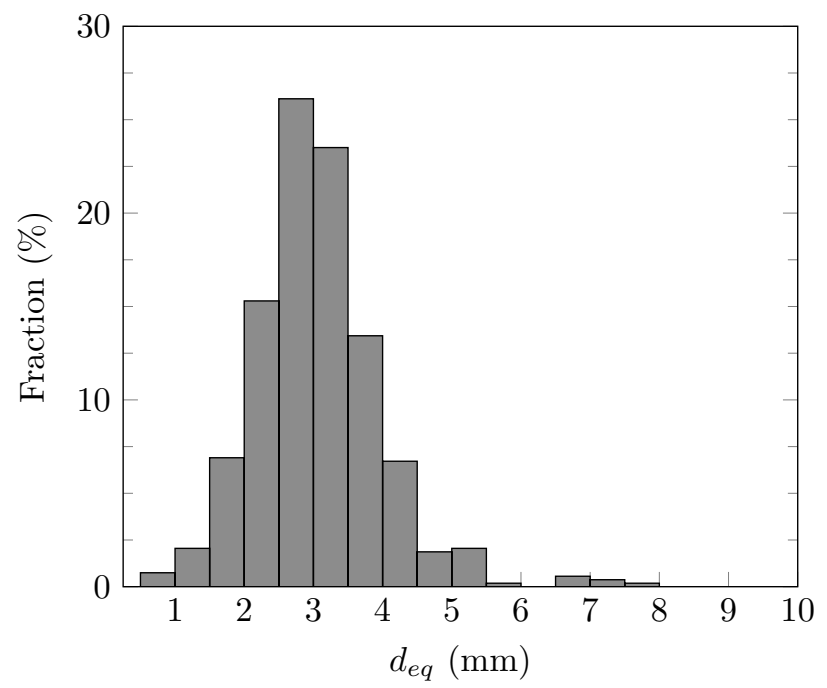

Figure 3: Equivalent diameters distribution. Total chip count: 536

As a result, one can consider that the batch of wood chips used for these experiments has an equivalent diameter $\left(\overline{d_{e q}}\right)$ of $3.0 \times 10^{-3} \mathrm{~m}$ with a standard deviation of $0.9 \times 10^{-3} \mathrm{~m}$, based on 536 , three dimensions measurements.

\section{Experimental reference}

\subsection{Experimental apparatus}

The experimental apparatus developed for permeability measurement is quite simple. Figure 4 presents this experimental device. Basically, it boils down to a 5 meter high, $8.0 \times 10^{-2} \mathrm{~m}$ diameter tube with a grid $5.0 \times 10^{-2} \mathrm{~m}$ above its lower extremity. The bed height above the pressure sensor is 4.65 meters. The height was chosen as the highest available in oder 
to maximize pressure loss, hence pressure signal. The diameter was chosen in order to minimize wall-particle ordering effects. These effects ranges from up to 2 diameters for spheres [38] to less than 1 diameter for anisotropic solids [39], such as our wood chips. The fraction of cross sectional area impacted by these wall effects is estimated to be lower than $5 \%$. Air tightness of the tubing was verified before and after the experimental measurements. Furthermore, even under load no deformation of the tube due to bed static pressure was measured.

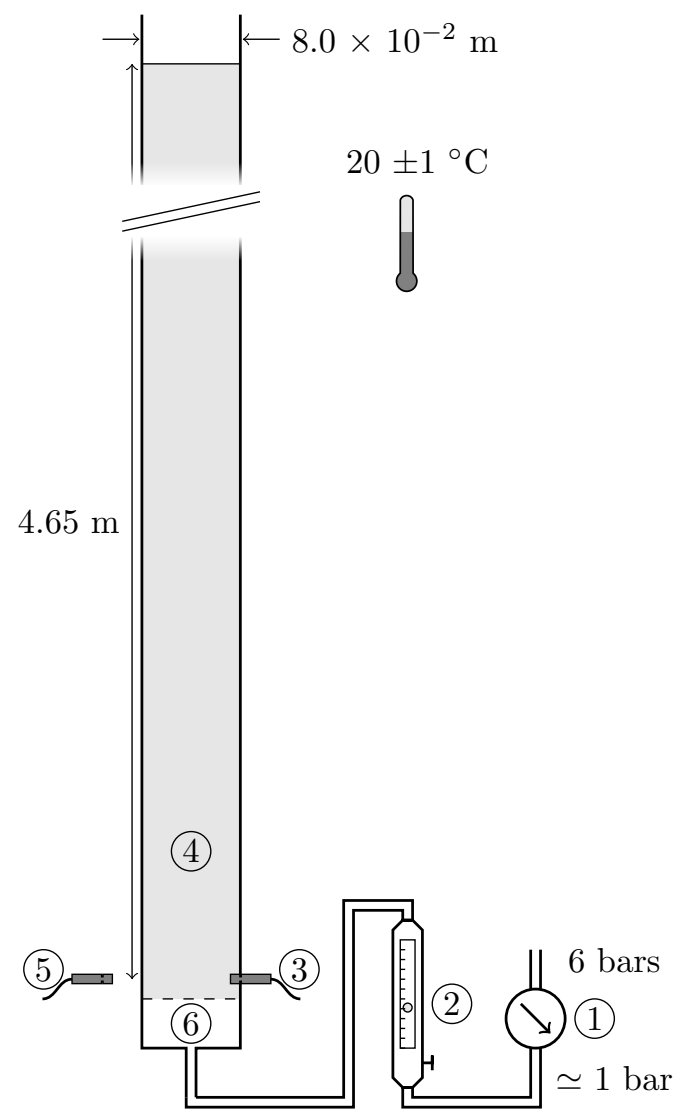

Figure 4: Experimental apparatus schematic. 1: pressure reducer, 2: flow controller, 3: bed pressure sensor, 4: wood chip bed, 5: room pressure sensor, 6: perforated grid $\left(10^{-3} \mathrm{~m}\right.$ diameter holes $)$

Both bed pressure and atmospheric pressure were monitored using RPS/DPS 8100 improved sensors, with 1 Pa resolution. Both sensors were set at the same height above the ground - one in the bed, the other out - in order to avoid any bias due to static pressure. The sensor monitoring the pressure at the bottom of the bed was placed $1.0 \times 10^{-2} \mathrm{~m}$ above the grid. The sensors were checked to yield the same values under room pressure before and after the experimental measurements. Even though the experiments took place in a temperature controlled room, air temperature was measured at the outlet of the tube using a thermocouple. The reported value is $20 \pm 1^{\circ} \mathrm{C}$.

Finally, in order to assess for experimental repeatability, experiments were conducted on two different beds. The tube was unloaded, chips were mixed, then the tube was reloaded. For both bed, pressure drops were measured over a range of flow rates, i.e. 10 to $100 \%$ of the flow controller range (Aalborg, precision $5 \%$, max. 15 1/min). As a consequence, Reynolds numbers range from 1 to 10 (Eq. 5). Provided no inertial effects are to be found in the openness between the chips, calculated permeabilities should be independent of the flow rate.

$$
R e=\frac{\rho \overline{d_{e q} Q}}{S \mu}
$$

\subsection{Experimental results}

Experiments were carried out successfully for both beds. Each pressure difference measurement was repeated three times. Deviation was below or equal to $1 \mathrm{~Pa}$. Hence, only averaged values are reported here. Pressure drops measurements and calculated permeabilities are available in Table 1. Pressure measurements are very close for both beds for Reynolds numbers between 2.14 and 5.35. Accordingly, calculated permeabilities are also quite close, with an average of $1.63 \times 10^{-8} \mathrm{~m}^{2}$. For a Reynolds number of 1.17, discrepancies emerge. They compensate one another and have no impact on the value of the permeability averaged over the different flow rates. For the highest Reynolds number (11.7), pressure drops dramatically 
increase, hence the permeabilities decrease. This in attributed to appearance of inertial effects inducing recirculations inbetween the chips in the bed. In this flow regime, Darcy's law is not suited anymore.

\begin{tabular}{ccccc}
\hline \multirow{2}{*}{ Re } & \multicolumn{2}{c}{ Pressure drop (Pa) } & \multicolumn{2}{c}{ Permeability $\left(\mathrm{m}^{2}\right)$} \\
& Bed 1 & Bed 2 & Bed 1 & Bed 2 \\
\hline 1.17 & 28 & 37 & $1.83 \times 10^{-8}$ & $1.40 \times 10^{-8}$ \\
2.33 & 63 & 63 & $1.64 \times 10^{-8}$ & $1.65 \times 10^{-8}$ \\
3.50 & 93 & 100 & $1.68 \times 10^{-8}$ & $1.56 \times 10^{-8}$ \\
5.83 & 160 & 160 & $1.62 \times 10^{-8}$ & $1.62 \times 10^{-8}$ \\
11.7 & 435 & 373 & $1.19 \times 10^{-8}$ & $1.39 \times 10^{-8}$ \\
\hline
\end{tabular}

Table 1: Experimentally measured pressure drops and calculated bed permeabilities for different Reynolds number

A point of note, with the aim of comparing these measurements to the numerical predictions, is the coefficient of variation of the measurements. Excluding the highest Reynolds number because the inertial effects, the coefficient of variation of the experimental measurements is $7.37 \%$. This has to be compared to the measurement uncertainty. By performing an uncertainty analysis, under random error assumption, the maximum uncertainty of our measurements was determined to be $8.2 \%$. The fact that the coefficient of variation is below the maximum uncertainty is a token of the quality of the experimental measurements. Going one step further and discarding the two extreme values, it falls down to $2.50 \%$. Hence, one can consider that the bed permeability value is $1.63 \pm 0.04 \times 10^{-8} \mathrm{~m}^{2}$.

The measured permeability values can be compared to the very widespread Kozeny-Carman expression [12], (Eq. 6). In this case, given the fact that woods chips cannot be assumed to be sphere. Indeed the population averaged aspect ratio (longest dimension divided by the shortest one) has a value of 5.4 (individual values ranging from $1.7 \mathrm{up}$ to 22 ). Hence, a correction factor $\Psi$ (Eq. 7) - the sphericity - is introduced. In order to evaluate this expression, sphericity was calculated for each of the 536 measured chips and then arithmetically averaged as $\bar{\Psi}=0.64$ (individual values ranging from 0.39 up to 0.79). Porosity also needs to be estimated. To do so, bed density was measured: $271 \pm 7 \mathrm{~kg} / \mathrm{m}^{3}$ (triplicates, filling a 1 liter beaker and weighting it). Beech wood chips density was measured with two methods: by weighting chips measured with a caliper and through infradensity measurements - weighting dry wood chips $\left(103.5^{\circ} \mathrm{C}, 24 \mathrm{~h}\right)$, impregnating them with water until mass stabilization, measuring saturated chips volume, correcting it using shrinkage measurements from literature [40] (established for millimeter size beech wood chips). Both methods yielded close values, $684 \mathrm{~kg} / \mathrm{m}^{3}$ for the first one, and 681 $\pm 6 \mathrm{~kg} / \mathrm{m}^{3}$ for the second. Close values have already been reported in the literature, in [41] the authors found a density of 680 for beech wood chips. Given the volume uncertainty associated with the first measurement method, the chips density was taken as $681 \mathrm{~kg} / \mathrm{m}^{3}$. Hence, the bed has a porosity of $0.60 \pm 0.02$, yielding a permeability of $3.44 \pm 0.52 \times 10^{-8} \mathrm{~m}^{2}$ according to Kozeny-Carman expression. This results calls for three comments. First, the predicted permeability is $115 \%$ away from the measured one, yet it provides the right order of magnitude. Second, even though the bed porosity is known with a very little uncertainty - coefficient of variation of $1.8 \%$-, the one on the permeability is quite high - coefficient of variation of 15.0 $\%$-. This last point originates from the high sensitivity of the Kozeny-Carman expression for porosities close to 0.5. It cannot be overcome. Third, Kozeny-Carman prediction could be further refine taking surface overlapping into account, which would lower the yielded permeability value. Yet, this process heavily relies on empirical, case dependent, studies [42, 43].

$$
\begin{gathered}
\kappa=\frac{{\overline{e_{e q}}}^{2} \bar{\Psi}^{2}}{150} \frac{\epsilon^{3}}{(1-\epsilon)^{2}} \\
\Psi=\frac{\pi^{1 / 3}(6 V)^{2 / 3}}{S}
\end{gathered}
$$

Finally, the computed values of permeability and porosity are in the vicinity of what was experimentally measured in the literature, for slightly larger wood chips $\left(\bar{d}_{e q}=3.7 \times 10^{-3} \mathrm{~m}\right.$, with a standard deviation of $\left.2.0 \times 10^{-3} \mathrm{~m}\right)[14]$.

\section{Numerical approach}

\subsection{Medium generation}

Obtaining a morphology of a large size particle bed can be a challenging task. Nevertheless, several alternatives are available: scanning a real bed (need for expensive apparatus, highly skilled staff, the scene may not even a large enough to be a Representative Elementary Volume - REV -), generating a medium using advanced mathematical functions [44](rising the question of reproduction of the actual spatial distribution inside of the bed), numerically computing particle-particle interactions when poured into the reactor, using a DEM code (such as LMGC90 [45] or LIGGGHTS [46]). This last method alleviate the difficulties of the former two. Furthermore, it has been successfully applied to mono-dispersed spheres [47, 48]. To be best of our knowledge, among the available DEM codes, only LMGC90 can properly handle polyhedron collision detection. This feature is an important step forward compared to current standard which uses agglomerated spheres to emulated polygons [49]. Hence, 
LMGC90 can properly compute friction stress and balance it with gravity. Figure 1 pictures actual chips and their numerical equivalent. Even though the reproduction is not entirely faithful, it remains very close to the original.

In order to reproduce the experimental conditions (Sec. 4.1), 15000 wood chips, randomly drawn from the 536 measured chips, were poured into a $8.0 \times 10^{-2} \mathrm{~m}$ diameter, $5 \mathrm{~m}$ high, tube. Physical parameters required for this computation are few, i.e. wood over wood friction coefficient $\zeta_{w-w}=0.56$ [50], wood over PVC friction coefficient $\zeta_{w-P V C}=0.44$ [50], wood density $681 \mathrm{~kg} / \mathrm{m}^{3}$ and acceleration due to gravity $|\vec{g}|=9.81 \mathrm{~m}^{2} / \mathrm{s}$. The calculations require about 4 days of computational time on a single thread (Intel Xeon CPU E5-2643 v3 @ 3.40GHz, RAM 64 Go $2133 \mathrm{MHz}$ ). Once completed, it is easy to extract a very light weight surface mesh that is water tight and therefore readily usable (Fig. 5).

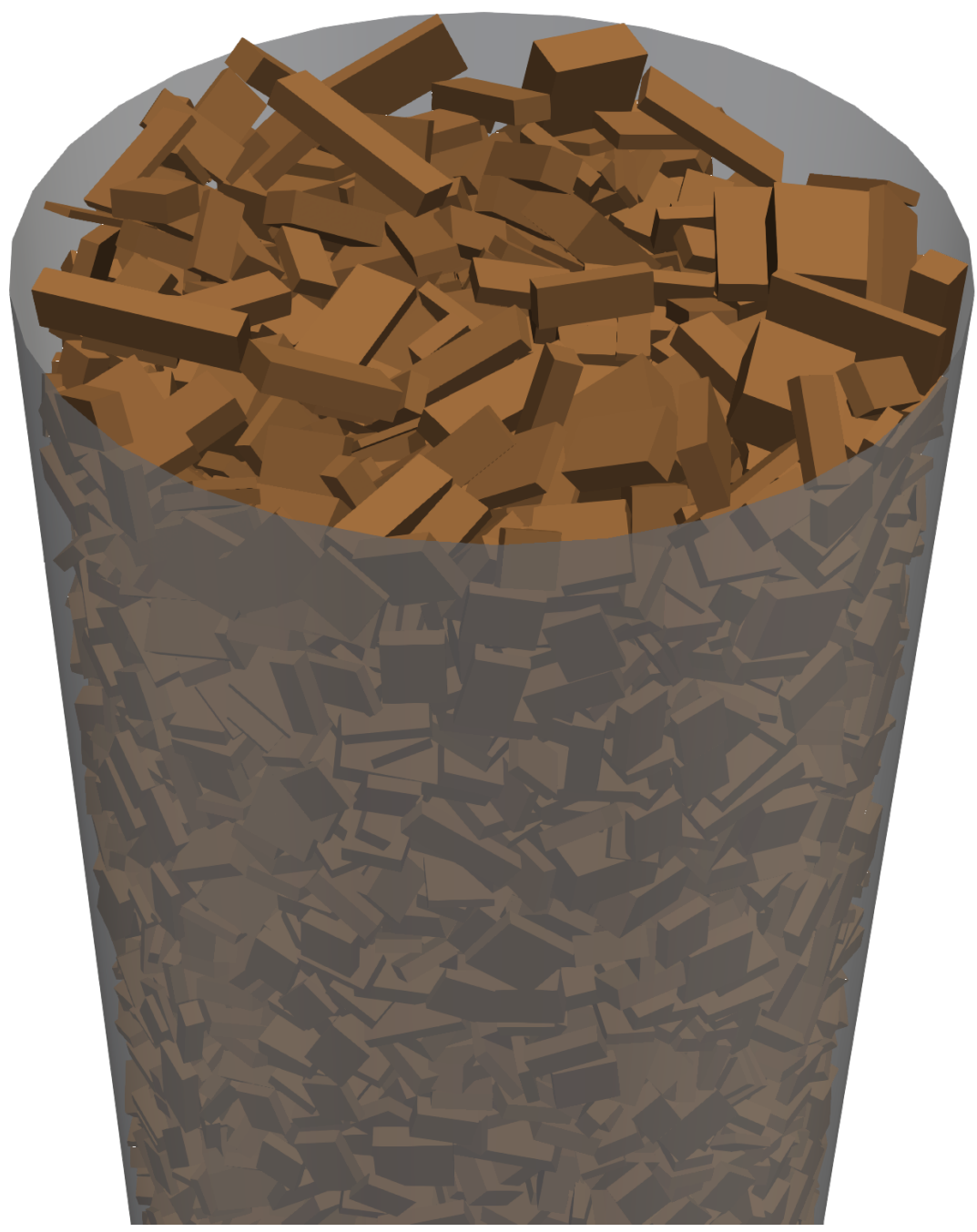

Figure 5: Top of the bed. Brown: wood chips, dark gray: tube

In order to assess for the repeatability of the process, three different beds (with different seeds for the random number generator) were produced.

\subsection{Computational domain meshing}

Before solving Navier-Stokes equations at the pore scale, the first step is to mesh the internal geometry of the computed bed. To do so, we used OpenFOAM CFD framework [51] (17.12+ release), as it also provides tools for meshing geometries. From this toolbox, we used the automatic meshing program called snappyHexMesh to mesh the fluid domain in-between the wood chips. This meshing algorithm works in three key stages (Fig. 6):

- first, a background mesh is generated. Then, wood chips surface are superimposed over the background mesh (Fig. 6a). The algorithm detects the cells that are intersected by the wood chips' surface and then divided in 8 (Fig. 6b). This 
creates a mesh refinement close to the body surface. This step is repeated a number of time prescribed by the operator (twice on Fig. 6c). Once this step is completed, cells that have less than $50 \%$ of their volume inside of the fluid domain are deleted. The number of consecutive divisions prescribed by the operator, as well as the initial mesh size, are key parameters whose influence is investigated in a coming Section (Sec. 5.4).

- then, the points of the mesh close the body surface are moved so that they snap onto it (Fig. 6d). This step ensures that the boundary surfaces of the mesh are as close as possible to the prescribed geometry.

- finally, three boundary layers of cells are added so that near wall effects are properly captured (on Fig. 6e, only one layer is drawn for illustrative purposes).

These steps are both CPU and RAM intensive, especially when the number of consecutive divisions prescribed in the first stage is high. To face the need for computational power, we used part the ROMEO cluster - 8 nodes, 16 cores (Intel Ivy Bridge $@ 2,6 \mathrm{GHz}$ ) and 32 Go DDR3 per node -.

\subsection{Fluid flow computation}

Fluid flows were calculated using Navier-Stokes equations in steady state, with no body force applied (hence, $\vec{f}=\overrightarrow{0}$ ), under incompressible flow assumption (Eq. 8 and 9). For turbulent flows, the stress tensor was modeled using Launder-Reece-Rodi model [52]. This turbulence model belong to the Reynolds Stress Model family. They describe turbulence using one equation for each of the Reynolds stress. They are far more complex than usual k- $\epsilon$ models, but they much better describe fluid-walls interactions. We used this model because accurately capturing those interactions is key in our case as the pressure drop originates form them.

$$
\nabla \cdot \rho \vec{u}=0
$$

$$
\frac{\partial \rho \vec{u}}{\partial t}+\nabla \cdot(\partial \rho \vec{u} \vec{u})=-\nabla \frac{P}{\rho}+\nabla \cdot \overline{\bar{\Sigma}}+\vec{f}
$$

At the inlet, the total inflow is specified while the pressure is set freely by the solver, as a consequence of the pressure drop across the medium:

$$
\begin{gathered}
\vec{u} \cdot \vec{n}=-\frac{Q}{S_{i n}} \\
\nabla P . \vec{n}=0
\end{gathered}
$$

At the outlet, the fluid can leave the domain freely under atmospheric pressure condition:

$$
\begin{gathered}
\nabla \vec{u} \cdot \vec{n}=\overrightarrow{0} \\
P=0
\end{gathered}
$$

On the walls, no slip condition is applied to the fluid motion:

$$
\begin{gathered}
\vec{u}=\overrightarrow{0} \\
\nabla P . \vec{n}=0
\end{gathered}
$$

On the side of the computational domain, two different kind of boundary conditions can be applied: no flow or symmetry, the later assuming that the domain is a subset of an infinite periodic domain. In their work [22], the authors showed that this choice has a minor impact provided the mesh it sufficiently refined - which is thought to be the case here. Following their recommendations, we used symmetry boundary conditions for the lateral boundaries of the computational domain.

Air physical properties were computed at $20^{\circ} \mathrm{C}$, as experimentally reported (Table 2).

\begin{tabular}{ccccc}
\hline Symbol & Property & Value & Dimension & Note \\
\hline $\mathrm{M}$ & Molar mass & 28.96 & $\mathrm{~g} / \mathrm{mol}$ & {$[53]$} \\
$\rho$ & Density & 1.205 & $\mathrm{~kg} / \mathrm{m}^{3}$ & Calculated \\
$\mu$ & Dynamic viscosity & $1.857 \times 10^{-5}$ & $\mathrm{~Pa} . \mathrm{s}$ & {$[53]$} \\
\hline
\end{tabular}

Table 2: Air physical properties, at $20^{\circ} \mathrm{C}$ under $101325 \mathrm{~Pa}$

The computations were led using the open source CFD framework OpenFOAM. More specifically, the aforementioned equation set was solved with simpleFoam solver, which uses SIMPLE algorithm to compute a Navier-Stokes equations steady state solution. Second order schemes, with flux limiter, were used for spatial discretization. Convergence criteria for all solution were set at $10^{-6}$. 


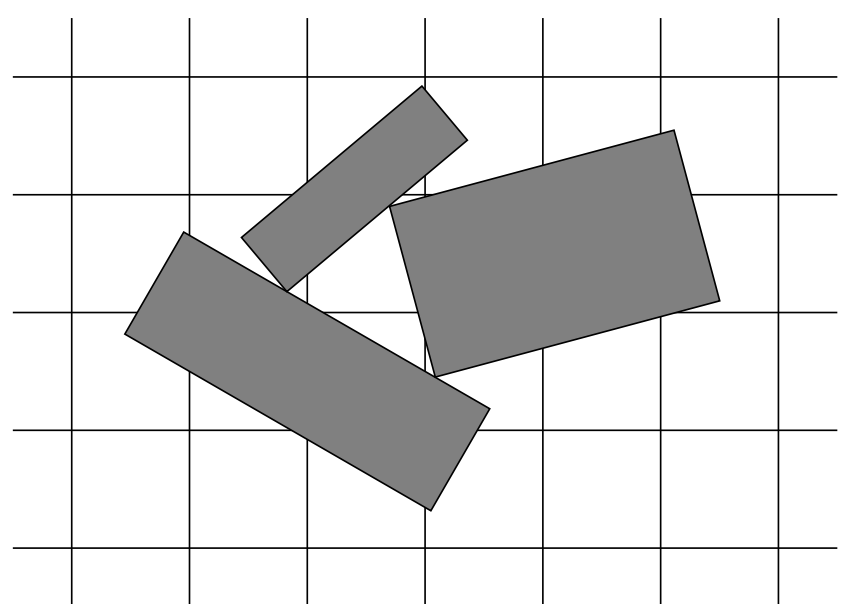

(a)

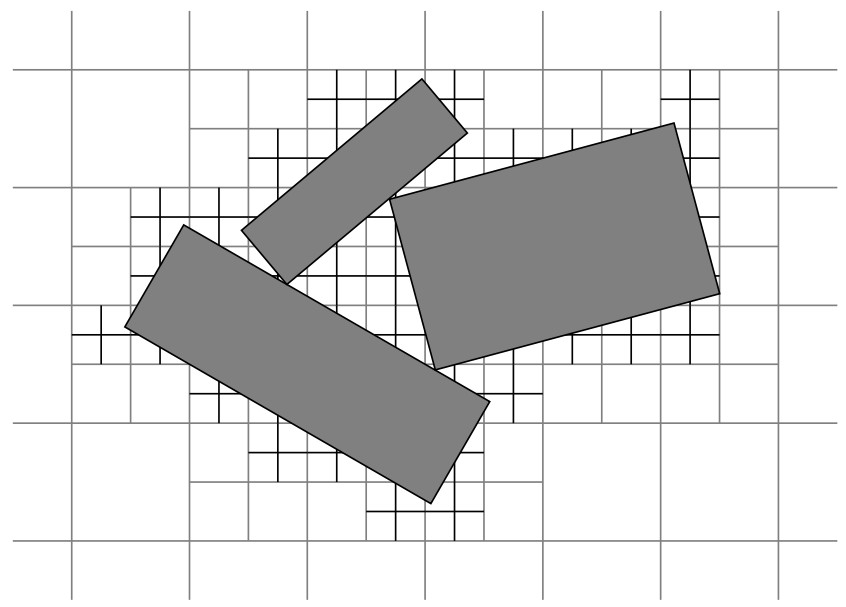

(c)

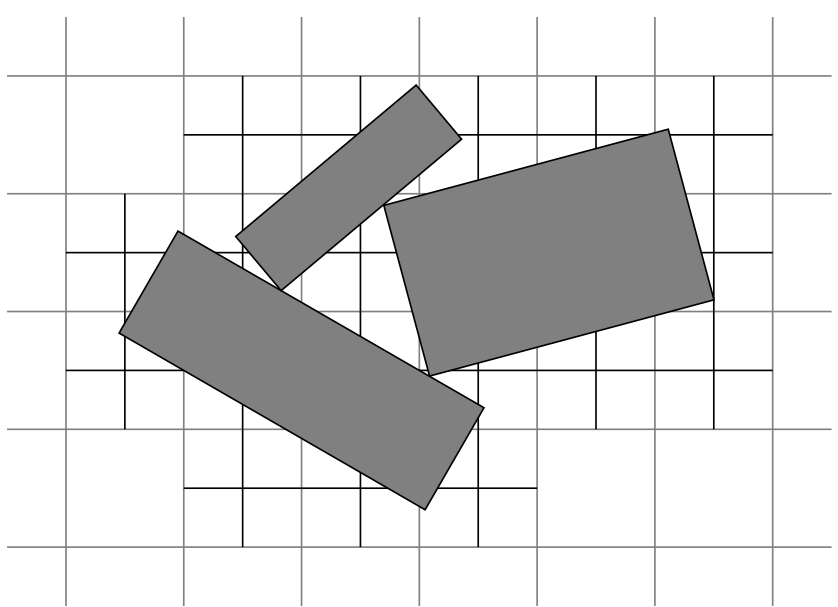

(b)

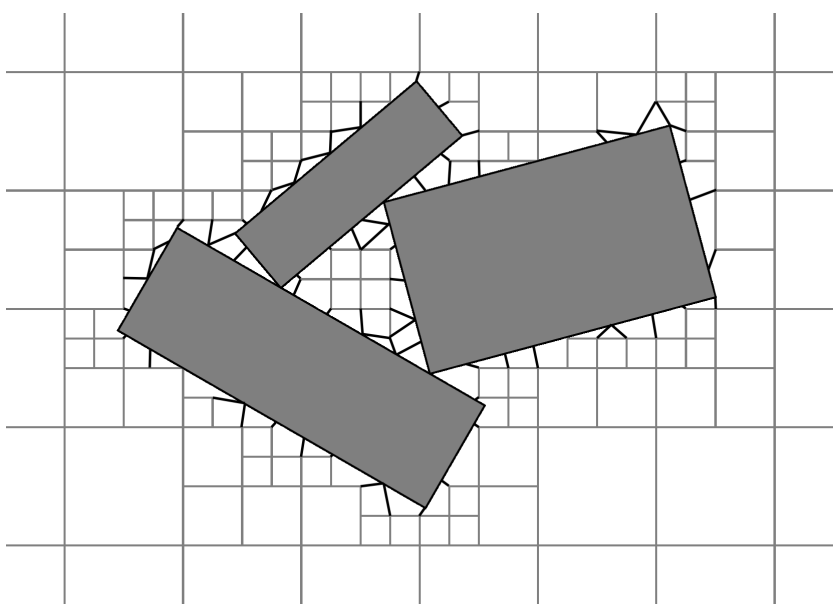

(d)

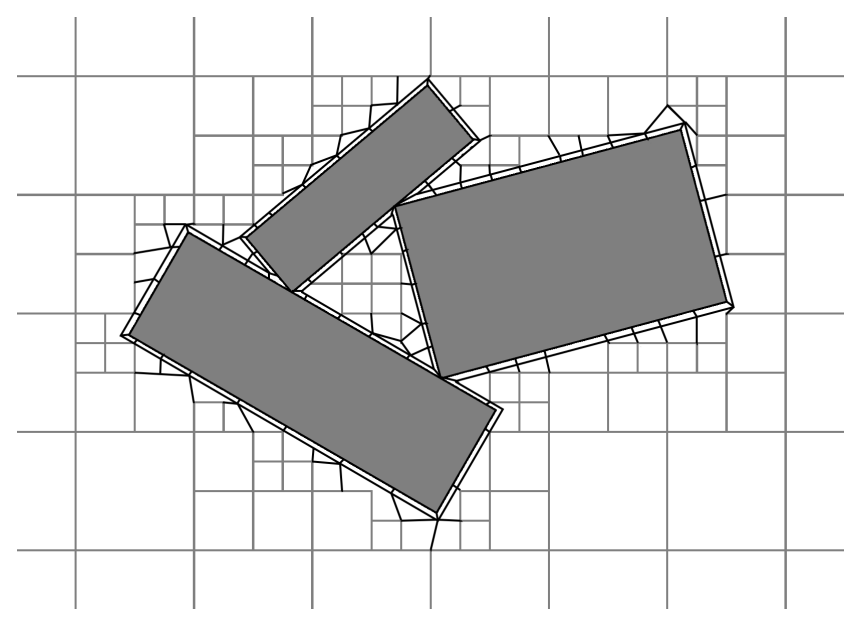

(e)

Figure 6: Meshing technique. (a) Background mesh and wood chips. (b) First refinement of the background mesh based of the intersection with the wood chips' surfaces. (c) Second refinement of the background mesh based of the intersection with the wood chips' surfaces. (d) Snapping of the refined mesh to the wood chips' surfaces. (e) Boundary layer addition 


\subsection{Mesh quality assessment}

Given the importance of number of consecutive divisions prescribed in the first of the meshing process and the intricacy of the geometry, it is mandatory to assess for the quality of the mesh. Indeed, a poor refinement level would yield grid dependent results, while an excessive refinement would lead to unnecessarily RAM intensive simulations or even not achievable computations.

The validation procedure was conducted on a small sample of the mesh ( $3 \overline{d_{e q}}$ side cube), with an initial mesh made of perfect $\overline{d_{e q}} / 3$ side hexahedrons. The number of consecutive divisions was varied from 0 to 9 . Three indicators were used to quantify the mesh quality: fluid phase volume (or bed porosity), wood chips surface area (or bed specific area) and pressure drop (with $\mathrm{Re}=0.05)$. Mesh porosity and specific area were confronted to reference values $\left(\mathrm{V}_{\text {ref }}\right.$ and $\left.\mathrm{S}_{\text {ref }}\right)$ known thanks to the fact that the medium has been generated numerically. Obtaining a reference value for pressure drop is not possible given the geometry. As a consequence, pressure drops were compared to the one computed for the most refined mesh.

Figure 7 reports the ratio between mesh volume and its reference value. As one can see, mesh volume is very close (less than $1 \%$ deviation) to the reference. This results if not surprising given the meshing approach. Yet, on closer inspection, it does not mean that the mesh is properly reproducing the geometry for low refinement values. Indeed, for refinement levels below 3, the closeness of the computed volume with its reference value can be explained by the fact that errors compensate one another. As a conclusion, mesh volume is a poor quality indicator.

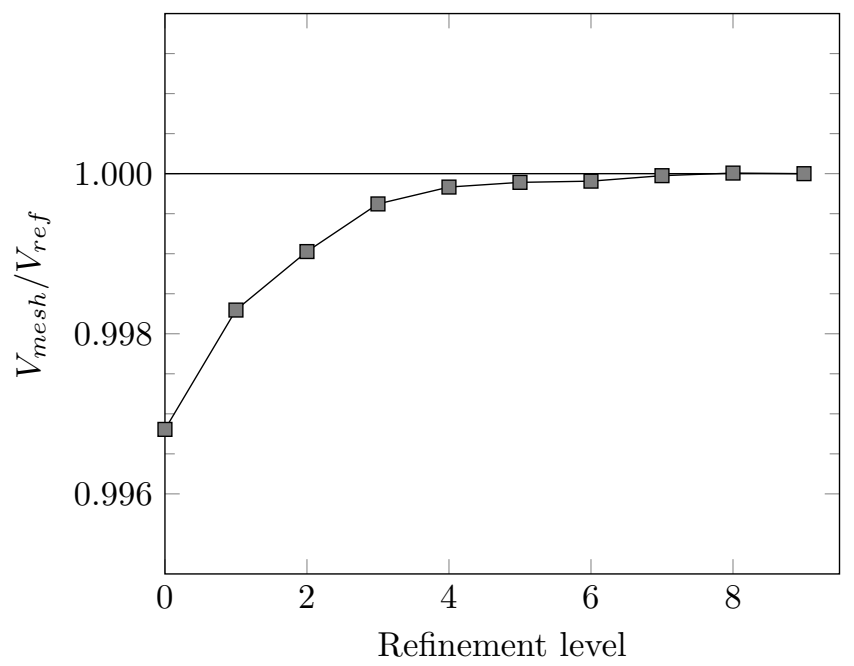

Figure 7: Mesh volume convergence with refinement level

Figure 8 shows the ratio between mesh wood chips surface area and its reference value. This ratio ranges from $72.5 \%$ to $96.5 \%$ for refinement levels of 0 and 9, respectively. Furthermore, at least 7 consecutive divisions of the background mesh are required to capture at least $90 \%$ of the actual surface area. By analyzing the areas of the geometry that the meshing algorithm fail to capture, one can realize that those parts of the are most intricated ones. As a first guess, given the shape of those parts of the geometry, they can only host very minor flows, if not at all. Thus, even though pressure drop is closely linked to the fluid-wall interactions, these areas may not contribute significantly to the total pressure drop.

Figure 9 presents the ratio between mesh pressure drop and its reference value. The value starts from $70 \%$, for an unrefined mesh, and rapidly convergence toward a stabilized value. Indeed, only 3 refinement levels are needed to reach $95 \%$ of the reference value, 5 consecutive divisions achieve $99 \%$ of the final value.

In this case of permeability computation, the most relevant indicator is the third one, i.e. the pressure drop. Owing respect to the results showed on Figure 9 and the uncertainty associated to an experimental measurement of a permeability $( \pm 2.50$ $\%$, see Sec. 4.2), we choose to work with a refinement level set at 4, i.e. a gap below $1.60 \%$ for the pressure drop.

\subsection{Computational domain size dependence}

Now that mesh quality as been assessed, it is possible to run proper simulations. A visualization of the flow inside of the bed can be found in Figure 10. Yet, before producing readily usable results, another important parameter has to be dealt with, i.e. computational domain size. Indeed, as porous media are prone to channeling, the computational domain should be large enough so that the results are not affected by this phenomenon.

To do so, the numerically generated beds were sampled with increasing volumes. For each volume, a full simulation was run. The flow had a Reynolds number of 0.05 to avoid inertial effects was would undermine the comparison. In order to assess for the repeatability of the numerical results, the sampling volumes were taken in the heart of the three beds at a height at least $20 \overline{d_{e q}}$ above the bottom and below the top. In addition, the first bed was sampled at two different heights (position 1: 


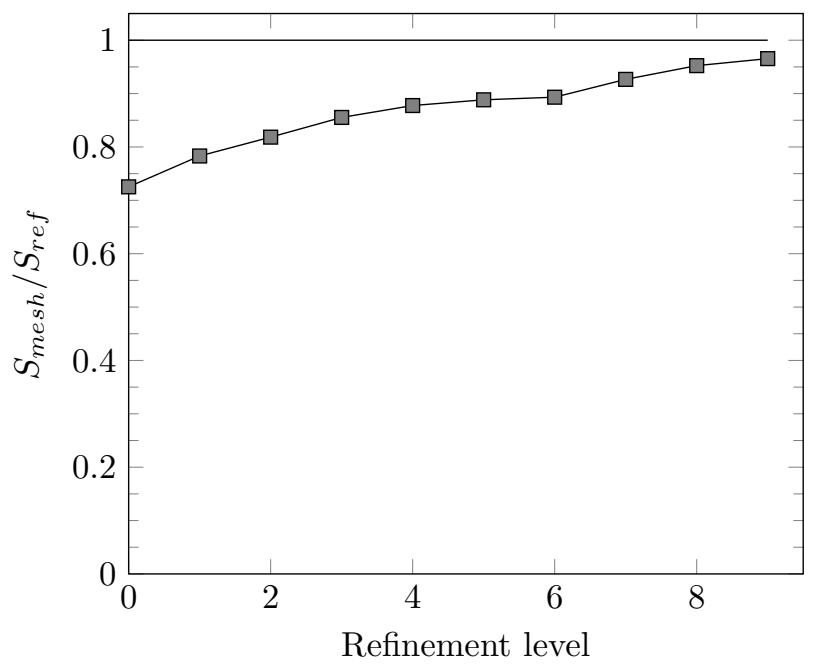

Figure 8: Mesh surface convergence with refinement level

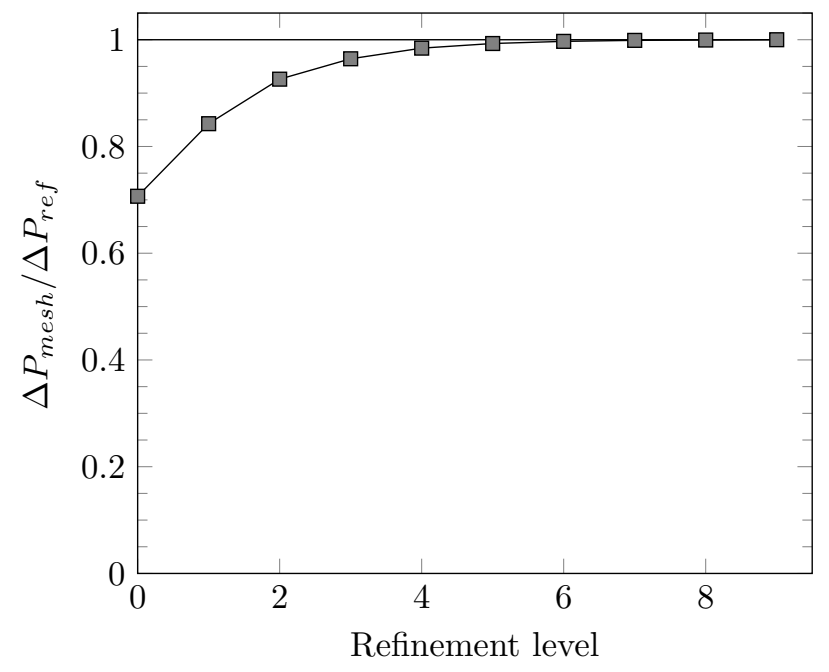

Figure 9: Pressure drop convergence with refinement level

$20 \overline{d_{e q}}$ above the bottom, position 2: $40 \overline{d_{e q}}$ above the bottom). These precautions ensure that the predictions do not depend on the numerically generated bed nor the sampling position.

Results are plotted on Figure 11. As one can see, computed permeabilities wary widely before stabilizing for computational domains larger than $11 \overline{d_{e q}}$. By averaging the results for simulations run with a sampling volume higher than $11 \overline{d_{e q}}$, the obtained permeability is $1.89 \pm 0.10 \times 10^{-8} \mathrm{~m}^{2}$. With a deviation of $16.0 \%$ - with reference to the experimental measurements - and a variation coefficient of $5.04 \%$, this result is much closer to reality and less dispersed than the Kozeny-Carman estimation.

The run time on the ROMEO cluster for the largest sampling volume was below two hours. Yet, one should keep in mind that with a Reynolds number of 0.05 , no turbulence model had to be solved.

Yet, these results call for discussion. Experimental measurements take into account wall effects, i.e. the potential channeling increasing the bed permeability. Even though these effects can be considered as minor, one could have expected the numerical workflow to yield a permeability value lower than the experimental one. In order to go one step further, numerical porosity was investigated. The numerical bed has a porosity of 0.47 , which is lower than the experimental value of 0.60 . This discrepancy could be explained by the role of two different factors: uncertainty associated to the wood-wood friction factor values and geometrical considerations. In order to investigate the first one, additional beds were generated with lower and higher friction factor values ( 0.46 and 0.66 , respectively). The resulting change in bed porosity was only marginal, with porosities of 0.46 and 0.48 , respectively. Hence, unknown over the friction factor value is not a valid explanation. The second explanation is based on the fact that the parallelepipedic description of the wood chips overestimates the chips volume. Yet again, intuitively, a lower porosity should lead to a lower permeability. Explaining this counterintuitive informations requires to go one step further and consider the origins of the pressure drop in this configuration. Permeability is measured in the Stokes regime, where pressure drop originates from viscous stress. Hence, the specific surface area is the physical property that determines the pressure drop. 


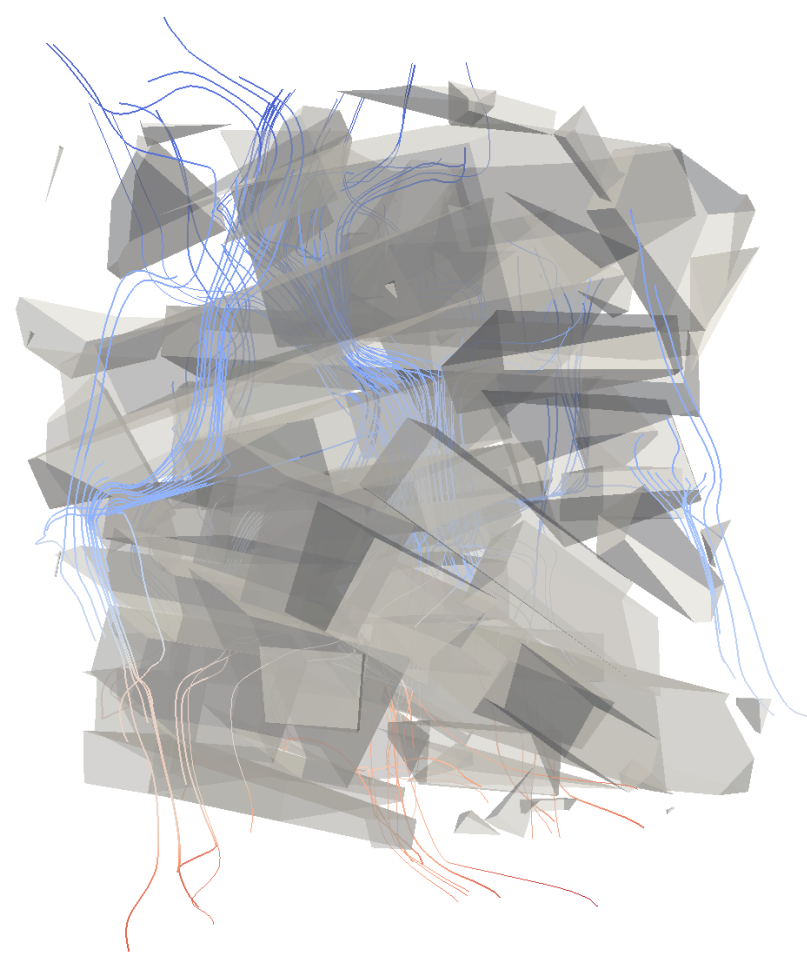

Figure 10: Upright flow visualization. Translucent gray: wood chips, colored lines: streamlines colored by pressure field values (seeds, two perpendicular horizontal lines crossing at the center of the sample)

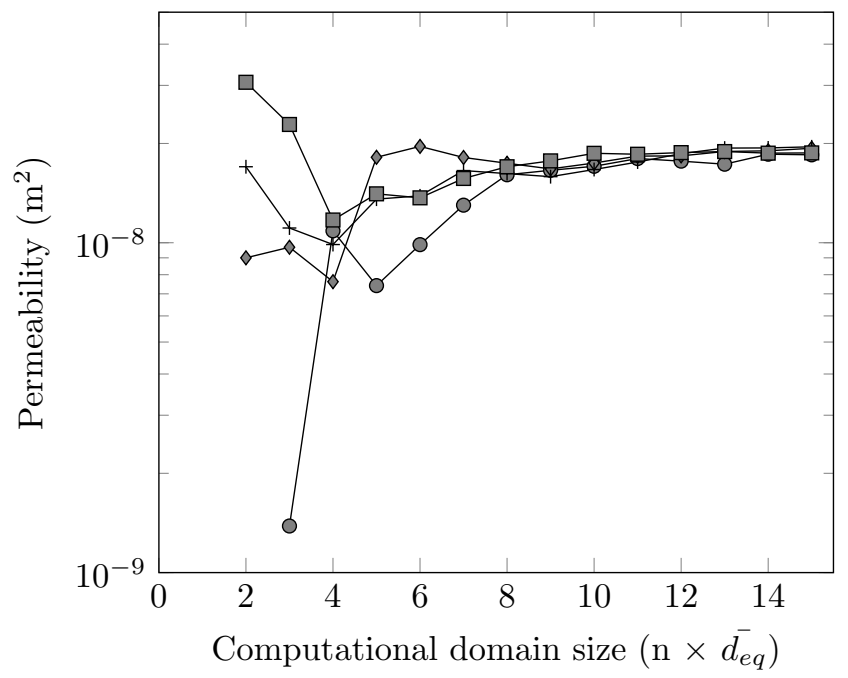

Figure 11: Pressure drop versus computational domain size. Squares: bed 1 at position 1, diamonds: bed 1 at position 2, circles: bed 2, crosses: bed 3

By using a parallelepipedic description of the wood chips, one overestimates the chip volume and underestimates the chip surface, thus the bed specific area. Furthermore, going from the real chips to their parallelepipedic counterparts also changes the packing geometry, which could explain the larger porosity of the numerical beds. Keeping in mind the huge influence of the porosity on permeability, to have such a good prediction is a real success. It indicates that, in spite of the simplification regarding the particles shapes, the digital bed generation captures accurately the morphological effects of the fluid pathway, ie. tortuosity and smallest sections along the pathways. 


\subsection{Permeability dependency on Reynolds number}

Using the developed workflow, it is possible to go one step further and compute pressure drop in the inertial regime. Indeed, most of the time industrial wood chips bed are operated in this flow regime. To do so, a sample domain of $12 \overline{d_{e q}}$ was taken out of the first bed. Then, simulations with a Reynolds number increasing for 0.01 to 1000 were run.

Figure 12 reports the evolution of the pressure gradient $\left(\frac{\Delta P}{h}\right)$ with the Reynolds number. A keen eye would notice two different trends on the graph: below a Reynolds number of about 2, the pressure gradient increases linearly; and above this value, the pressure gradient has quadratic dependency on Reynolds number. For the sake of readability, these results are also presented in terms of computed permeability on Figure 13. On this last graph, the transition between the zones appears clearly.

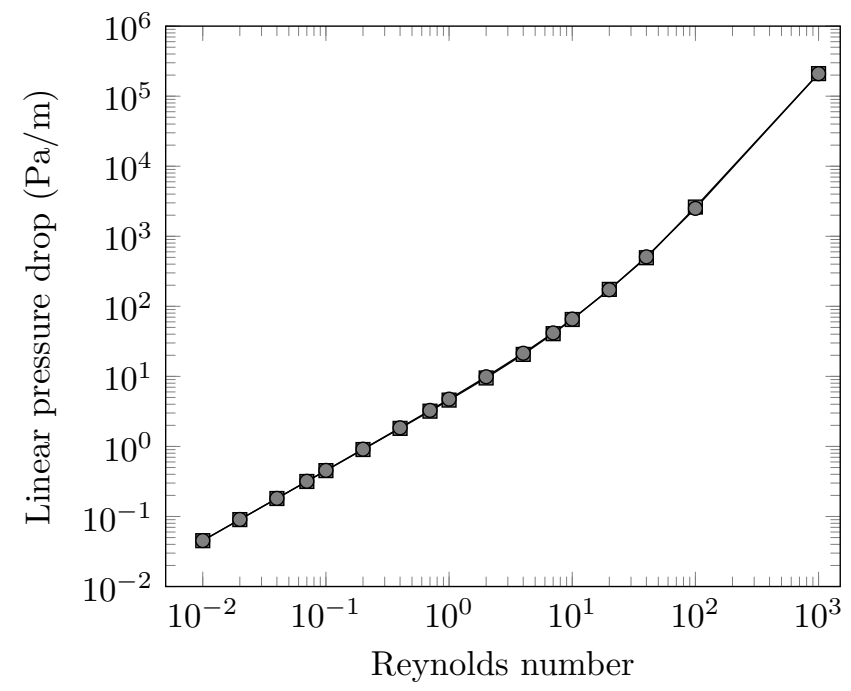

Figure 12: Pressure gradient evolution with increasing Reynolds number

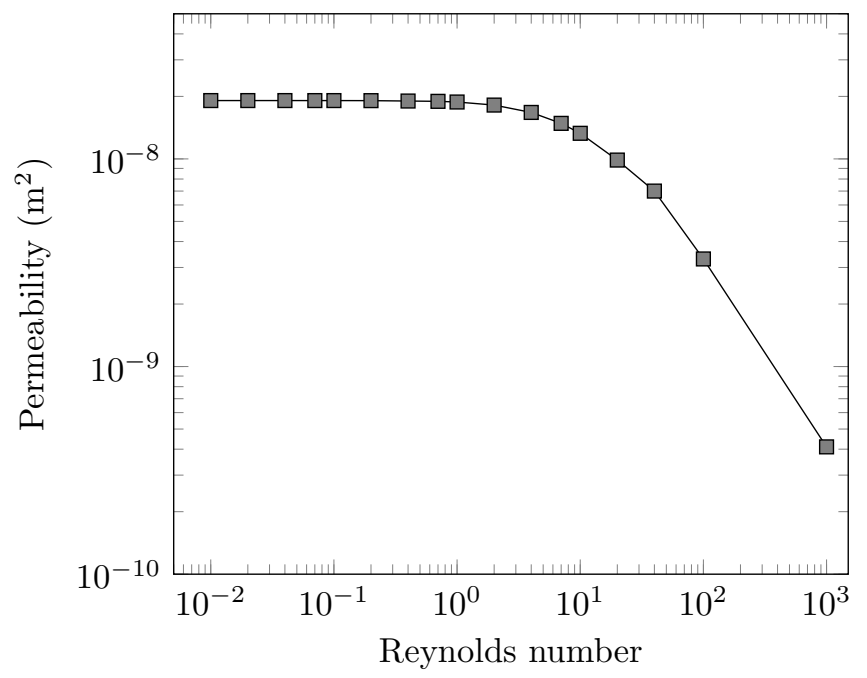

Figure 13: Computed permeability evolution with increasing Reynolds number

The quadratic dependency of the pressure gradient in the inertial zone is a very well established fact. As aforementioned, several correlations exist. In the community of biomass transformation, Forshheimer equation is the reference (Eq. 16). In this equation, the pressure gradient has two components, the first one $\left(\frac{\mu}{\kappa}|\vec{u}|\right)$ originating form Darcy's law and describing the flow in Stokes regime, the second one $\left(\rho C|\vec{u}|^{2}\right)$ accounting for inertial effects. In the second member of the equation, the constant $C$ is the key parameter. In our case, it was fitted versus the numerical data produced using the workflow. We obtained a value of $C=7865$ (average error $1.20 \%$ ). This value can vary widely depending on the medium, yet, in our case, it in good agreement with previously reported values, 6440 for unsorted $\overline{d_{e q}}=3.7 \times 10^{-3} \mathrm{~m}$ wood chips [14]. Ergun equation (Eq. 17) is also well-established in the chemical engineering community. Compared to the Forshheimer equation, it explicitly states 
the role of the porosity in both Stokes and inertial regimes. In our case, the fitting factor for the Stokes regime, referred as $\alpha$, has a value of 284 while 150 is classically used for monodispersed spheres. Furthermore, the numerical workflow yielded a value of 2.92 for fitting factor in the inertial regime, referred as $\beta$, while the commonly used value for monodispersed spheres is 1.75. Both factors are in the same order of magnitude as the their counterparts for monodispersed spheres. One could even note that the discrepancies are quite low given the shape difference between chips and spheres. This shows that the proposed method agrees well with established literature.

Furthermore, when applied to the prediction of the pressure drop for the $R e=11.7$ case, this inertial correction yields a value of $434 \mathrm{~Pa}$. This value is in agreement with experimental measurement (Tab. 1). Furthermore, the calculated value belongs to the upper range of experimental observations. This can be linked to the parallelepipedic description of the wood chips. In the same way as it may reduce bed specific area, hence the increase the permeability, it also reduces the porosity by virtually enlarging the bodies. In the inertial regime, the pressure drop originates from the bodies' shapes, hence overestimating this shape may lead to an overestimation of the pressure drop. As a results, predictions could be further improved by a better reconstruction of the chips, by taking trapezoidal shapes for example.

$$
\begin{gathered}
\frac{\Delta P}{h}=\frac{\mu}{\kappa}|\vec{u}|+\rho C|\vec{u}|^{2} \\
\frac{\Delta P}{h}=\alpha \frac{(1-\epsilon)^{2}}{{\overline{d_{e q}}}^{2} \bar{\Psi}^{2} \epsilon^{3}} \mu|\vec{u}|+\beta \rho|\vec{u}|^{2} \frac{1-\epsilon}{\overline{d_{e q} \bar{\Psi} \epsilon^{3}}}
\end{gathered}
$$

\section{Conclusion}

This work presented a numerical worklflow allowing the permeability of a granular packed bed made of non-spherical polydisperse particles to be computed using the particle size distribution as sole input data. The virtual packed bed is then generated using a DEM code, meshed and finally simulated in both Stokes and inertial regimes. Throughout this article, a special care has been taken in explaining and evaluating the impact of all the key parameters, namely, number of particles that have to be sized, mesh refinement level, numerical domain dimensions.

Experimental were performed on real packed beds to assess for the reliability of the workflow. The permeability value delivered by the workflow is $16.0 \%$ higher than the experimental value. This can be considered a major improvement over Kozeny-Carman equation, even corrected for non-spherical particles, which overestimates bed permeability by $115 \%$. Considering the proposed method as validated, this framework was successfully used to compute inertial effects constant of the Forshheimer equation for our packed bed.

All in all, this approach paves to way to the evaluation of bed tortuosity, dispersion coefficients, volumetric heat exchange coefficients, and much more, from fully virtual packed bed.

\section{Acknowledgements}

The authors would like to thank Département de la Marne, Région Grand Est, and Grand Reims for their financial support. This work was partially supported by the French HPC Center ROMEO.

\section{References}

[1] Y. Ding, Y. He, N. T. Cong, W. Yang, H. Chen, Hydrodynamics and heat transfer of gas-solid two-phase mixtures flowing through packed beds - a review, Progress in Natural Science-Materials International 18 (10) (2008) 1185-1196, wOS:000260324100001. doi:10.1016/j.pnsc.2008.03.023.

[2] S. P. Singh, P. Prerna, Review of recent advances in anaerobic packed-bed biogas reactors, Renewable \& Sustainable Energy Reviews 13 (6-7) (2009) 1569-1575, wOS:000266019300035. doi:10.1016/j.rser.2008.08.006.

[3] C. Noubactep, Designing Metallic Iron Packed-Beds for Water Treatment: A Critical Review, Clean-Soil Air Water 44 (4) (2016) 411-421, wOS:000374541500011. doi:10.1002/clen.201400304.

[4] S. K. Yeboah, J. Darkwa, A critical review of thermal enhancement of packed beds for water vapour adsorption, Renewable \& Sustainable Energy Reviews 58 (2016) 1500-1520, wOS:000371948100124. doi:10.1016/j.rser.2015.12.134.

[5] G. Aparicio-Mauricio, R. S. Ruiz, F. Lopez-Isunza, C. O. Castillo-Araiza, A simple approach to describe hydrodynamics and its effect on heat and mass transport in an industrial wall-cooled fixed bed catalytic reactor: ODH of ethane on a MoVNbTeO formulation, Chemical Engineering Journal 321 (2017) 584-599, wOS:000401041900057. doi:10.1016/j.cej.2017.03.043.

[6] A. Faridkhou, M. Hamidipour, F. Larachi, Hydrodynamics of gas-liquid micro-fixed beds - Measurement approaches and technical challenges, Chemical Engineering Journal 223 (2013) 425-435, wOS:000320631200049. doi:10.1016/j.cej.2013.03.014. 
[7] A. Faridkhou, F. Larachi, Two-phase flow hydrodynamic study in micro-packed beds - Effect of bed geometry and particle size, Chemical Engineering and Processing 78 (2014) 27-36, wOS:000335625600004. doi:10.1016/j.cep.2014.02.005.

[8] I. Iliuta, F. C. Thyrion, L. Bolle, M. Giot, Comparison of hydrodynamic parameters for countercurrent and cocurrent flow through packed beds, Chemical Engineering \& Technology 20 (3) (1997) 171-181, wOS:A1997XA84100003. doi:10.1002/ceat.270200304.

[9] N. Al-Rifai, F. Galvanin, M. Morad, E. Cao, S. Cattaneo, M. Sankar, V. Dua, G. Hutchings, A. Gavriilidis, Hydrodynamic effects on three phase micro-packed bed reactor performance - Gold-palladium catalysed benzyl alcohol oxidation, Chemical Engineering Science 149 (2016) 129-142, wOS:000376522600013. doi:10.1016/j.ces.2016.03.018.

[10] P. Agrawal, A. Gautam, A. Kunwar, M. Kumar, S. Chamoli, Performance assessment of heat transfer and friction characteristics of a packed bed heat storage system embedded with internal grooved cylinders, Solar Energy 161 (2018) 148-158. doi:10.1016/j.solener.2017.12.044.

URL http://www.sciencedirect.com/science/article/pii/S0038092X1731126X

[11] D. A. Nield, A. Bejan, Convection in Porous Media, 4th Edition, Springer-Verlag, New York, 2013. URL //www. springer. com/gp/book/9781489998224

[12] P. C. Carman, Flow of gases through porous media. Butterworths, London., Flow of gases through porous media. Butterworths, London. (1956) -.

[13] H. Darcy, The public fountains of the city of Dijon, Victor Dalmont, Paris, France.

[14] M. Mayerhofer, J. Govaerts, N. Parmentier, H. Jeanmart, L. Helsen, Experimental investigation of pressure drop in packed beds of irregular shaped wood particles, Powder Technology 205 (1) (2011) 30-35. doi:10.1016/j.powtec.2010.08.006. URL http://www.sciencedirect.com/science/article/pii/S0032591010003980

[15] K. Ueda, M. Fukuoka, N. Yoshioka, Permeability of Beds Packed with Permeable and Porous Spheres, Kagaku Kogaku Ronbunshu 9 (4) (1983) 465-467, wOS:A1983QY85400018.

[16] Y. C. Li, C. W. Park, Permeability of packed beds filled with polydisperse spherical particles, Industrial \& Engineering Chemistry Research 37 (5) (1998) 2005-2011, wOS:000073501200055. doi:10.1021/ie970603s.

[17] T. C. S. Pinto, O. A. Lima, L. S. Leal Filho, Sphericity of apatite particles determined by gas permeability through packed beds, Minerals \& Metallurgical Processing 26 (2) (2009) 105-108, wOS:000269142700008.

[18] B. R. Corrochano, J. R. Melrose, A. C. Bentley, P. J. Fryer, S. Bakalis, A new methodology to estimate the steadystate permeability of roast and ground coffee in packed beds, Journal of Food Engineering 150 (2015) 106-116, wOS:000349062600014. doi:10.1016/j.jfoodeng.2014.11.006.

[19] R. Sander, Z. Pan, L. D. Connell, Laboratory measurement of low permeability unconventional gas reservoir rocks: A review of experimental methods, Journal of Natural Gas Science and Engineering 37 (2017) 248-279, wOS:000392679900018. doi:10.1016/j.jngse.2016.11.041.

[20] A. L. Redman, H. Bailleres, I. Turner, P. Perré, MASS TRANSFER PROPERTIES (PERMEABILITY AND MASS DIFFUSIVITY) OF FOUR AUSTRALIAN HARDWOOD SPECIES, BioResources 7 (3) (2012) 3410-3424.

URL http://www.ncsu.edu/bioresources/BioRes_07/BioRes_07_3_3410_Redman_BTP_Mass_Trans_Prop_Austral_HW_ Spp_2648.pdf

[21] H. Lee, S. Koo, Liquid permeability of packed bed with binary mixture of particles, Journal of Industrial and Engineering Chemistry 20 (4) (2014) 1397-1401, wOS:000337775100035. doi:10.1016/j.jiec.2013.07.024.

[22] R. Guibert, M. Nazarova, P. Horgue, G. Hamon, P. Creux, G. Debenest, Computational Permeability Determination from Pore-Scale Imaging: Sample Size, Mesh and Method Sensitivities, Transport in Porous Media 107 (3) (2015) $641-656$. doi:10.1007/s11242-015-0458-0.

URL https://link.springer.com/article/10.1007/s11242-015-0458-0

[23] P. Ranut, E. Nobile, L. Mancini, High resolution X-ray microtomography-based CFD simulation for the characterization of flow permeability and effective thermal conductivity of aluminum metal foams, Experimental Thermal and Fluid Science 67 (2015) 30-36. doi:10.1016/j.expthermflusci.2014.10.018.

URL http://www.sciencedirect.com/science/article/pii/S089417771400260X

[24] S. Jaganathan, H. Vahedi Tafreshi, B. Pourdeyhimi, A realistic approach for modeling permeability of fibrous media: 3-D imaging coupled with CFD simulation, Chemical Engineering Science 63 (1) (2008) 244-252. doi:10.1016/j.ces.2007.09.020. URL http://www.sciencedirect.com/science/article/pii/S0009250907007312

[25] R. Guibert, P. Horgue, G. Debenest, M. Quintard, A Comparison of Various Methods for the Numerical Evaluation of Porous Media Permeability Tensors from Pore-Scale Geometry, Mathematical Geosciences 48 (3) (2016) $329-347$. doi:10.1007/s11004-015-9587-9.

URL https://link.springer.com/article/10.1007/s11004-015-9587-9

[26] S. K. Singh, M. Kumar, A. Kumar, A. Gautam, S. Chamoli, Thermal and friction characteristics of a circular tube fitted with perforated hollow circular cylinder inserts, Applied Thermal Engineering 130 (2018) $230-241$. doi:10.1016/j.applthermaleng.2017.10.090.

URL http://www.sciencedirect.com/science/article/pii/S1359431117337821 
[27] S. Chamoli, R. Lu, D. Xu, P. Yu, Thermal performance improvement of a solar air heater fitted with winglet vortex generators, Solar Energy 159 (2018) 966-983. doi:10.1016/j.solener.2017.11.046.

URL http://www.sciencedirect.com/science/article/pii/S0038092X17310381

[28] S. Chamoli, R. Lu, P. Yu, Thermal characteristic of a turbulent flow through a circular tube fitted with perforated vortex generator inserts, Applied Thermal Engineering 121 (2017) 1117-1134. doi:10.1016/j.applthermaleng.2017.03.145. URL http://www.sciencedirect.com/science/article/pii/S1359431117305264

[29] S. Chamoli, R. Lu, J. Xie, P. Yu, Numerical study on flow structure and heat transfer in a circular tube integrated with novel anchor shaped inserts, Applied Thermal Engineering 135 (2018) 304-324. doi:10.1016/j.applthermaleng.2018.02.052. URL http://www.sciencedirect.com/science/article/pii/S1359431117367522

[30] S. Jegatheeswaran, F. Ein-Mozaffari, J. Wu, Process intensification in a chaotic SMX static mixer to achieve an energyefficient mixing operation of non-newtonian fluids, Chemical Engineering and Processing - Process Intensification 124 (2018) 1-10. doi:10.1016/j.cep.2017.11.018.

URL http://www.sciencedirect.com/science/article/pii/S0255270117307031

[31] E. Gordillo, A. Belghit, A downdraft high temperature steam-only solar gasifier of biomass char: A modelling study, Biomass and Bioenergy 35 (5) (2011) 2034-2043. doi:10.1016/j.biombioe.2011.01.051.

URL http://www.sciencedirect.com/science/article/pii/S0961953411000663

[32] N. Piatkowski, A. Steinfeld, Solar-driven coal gasification in a thermally irradiated packed-bed reactor, Energy \& Fuels 22 (3) (2008) 2043-2052, wOS:000256057600086. doi:10.1021/ef800027c.

[33] S. Chopra, A. Jain, A review of Fixed Bed Gasification Systems for Biomass. URL http://ecommons. cornell.edu/handle/1813/10671

[34] Q. Zhang, J. Chang, T. Wang, Y. Xu, Review of biomass pyrolysis oil properties and upgrading research, Energy Conversion and Management 48 (1) (2007) 87-92. doi:10.1016/j.enconman.2006.05.010.

URL http://www.sciencedirect.com/science/article/pii/S0196890406001701

[35] H. Hartmann, T. Böhm, P. Daugbjerg Jensen, M. Temmerman, F. Rabier, M. Golser, Methods for size classification of wood chips, Biomass and Bioenergy 30 (11) (2006) 944-953. doi:10.1016/j.biombioe.2006.06.010.

URL http://www.sciencedirect.com/science/article/pii/S0961953406001000

[36] T. Shinbrot, Granular materials: The brazil nut effect — in reverse, Nature 429 (6990) (2004) 352-353. doi:10.1038/429352b.

URL https://www. nature.com/articles/429352b

[37] J. M. N. T. Gray, K. Hutter, Pattern formation in granular avalanches, Continuum Mechanics and Thermodynamics 9 (6) (1997) 341-345. doi:10.1007/s001610050075.

URL https://link.springer.com/article/10.1007/s001610050075

[38] S. M. White, P. C. L. Tien, Analysis of flow channeling near the wall in packed beds, Wärme - und Stoffübertragung 21 (5) (1987) 291-296. doi:10.1007/BF01009290.

URL http://link. springer.com/article/10.1007/BF01009290

[39] A. Montillet, L. Le Coq, Characteristics of fixed beds packed with anisotropic particles - Use of image analysis, Powder Technology 121 (2) (2001) 138-148. doi:10.1016/S0032-5910(01)00332-1.

URL http://www.sciencedirect.com/science/article/pii/S0032591001003321

[40] G. Almeida, C. Assor, P. Perré, The Dynamic of Shrinkage/Moisture Content Behavior Determined During Drying of Microsamples for Different Kinds of Wood, Drying Technology 26 (9) (2008) 1118-1124. doi:10.1080/07373930802266108. URL https://doi.org/10.1080/07373930802266108

[41] V. Repellin, A. Govin, M. Rolland, R. Guyonnet, Modelling anhydrous weight loss of wood chips during torrefaction in a pilot kiln, Biomass and Bioenergy 34 (5) (2010) 602-609. doi:10.1016/j.biombioe.2010.01.002.

URL http://www.sciencedirect.com/science/article/pii/S0961953410000036

[42] É. Trudel, W. Hallett, Pressure drop in packed beds of angular parallelepipeds, including the effects of particle interference, Chemical Engineering Science 162 (2017) 209-217. doi:10.1016/j.ces.2017.01.004.

URL http://www.sciencedirect.com/science/article/pii/S0009250917300209

[43] J. Comiti, M. Renaud, A new model for determining mean structure parameters of fixed beds from pressure drop measurements: application to beds packed with parallelepipedal particles, Chemical Engineering Science 44 (7) (1989) 1539-1545. doi:10.1016/0009-2509(89)80031-4.

URL http://www.sciencedirect.com/science/article/pii/0009250989800314

[44] S. Falco, F. De Cola, N. Petrinic, A method for the generation of 3d representative models of granular based materials, International Journal for Numerical Methods in Engineering 112 (4) (2017) 338-359, wOS:000411647100002. doi:10.1002/nme.5521.

[45] F. Dubois, M. Jean, M. Renouf, R. Mozul, A. Martin, M. Bagneris, Lmgc90, in: 10e colloque national en calcul des structures, 2011, p. Clé USB. 
[46] C. Kloss, C. Goniva, LIGGGHTS-open source discrete element simulations of granular materials based on Lammps, Supplemental Proceedings: Materials Fabrication, Properties, Characterization, and Modeling, Volume 2 (2011) $781-788$.

[47] W. Sobieski, Q. Zhang, C. Liu, Predicting Tortuosity for Airflow Through Porous Beds Consisting of Randomly Packed Spherical Particles, Transport in Porous Media 93 (3) (2012) 431-451. doi:10.1007/s11242-012-9961-8.

URL http://link. springer.com/article/10.1007/s11242-012-9961-8

[48] S. Das, N. G. Deen, J. A. M. Kuipers, A DNS study of flow and heat transfer through slender fixed-bed reactors randomly packed with spherical particles, Chemical Engineering Science 160 (2017) 1-19. doi:10.1016/j.ces.2016.11.008.

URL http://www.sciencedirect.com/science/article/pii/S0009250916306005

[49] T. J. Donohue, C. M. Wensrich, A numerical investigation of the void structure of fibrous materials, Powder Technology 186 (1) (2008) 72-79. doi:10.1016/j.powtec.2007.11.002.

URL http://www.sciencedirect.com/science/article/pii/S0032591007005608

[50] Y. Murase, Friction of Wood Sliding on Various Materials, Journal of the Faculty of Agriculture, Kyushu University 28 (4) (1984) $147-160$.

URL https://ci.nii.ac.jp/naid/110000017511/

[51] H. Jasak, A. Jemcov, Z. Tukovic, OpenFOAM: A C++ library for complex physics simulations, in: International workshop on coupled methods in numerical dynamics, Vol. 1000, IUC Dubrovnik, Croatia, 2007, pp. 1-20.

[52] B. E. Launder, G. J. Reece, W. Rodi, Progress in the development of a Reynolds-stress turbulence closure, Journal of Fluid Mechanics 68 (3) (1975) 537-566. doi:10.1017/S0022112075001814.

URL https://www.cambridge.org/core/journals/journal-of-fluid-mechanics/article/ progress-in-the-development-of-a-reynoldsstress-turbulence-closure/796DDAC14EF54A84A36100565D3420D5

[53] K. Kadoya, N. Matsunaga, A. Nagashima, Viscosity and Thermal Conductivity of Dry Air in the Gaseous Phase, Journal of Physical and Chemical Reference Data 14 (4) (1985) 947-970. doi:10.1063/1.555744.

URL https://aip.scitation.org/doi/10.1063/1.555744 